\title{
GPS: A General Peer-to-Peer Simulator and its Use for Modeling BitTorrent*
}

\author{
Weishuai Yang and Nael Abu-Ghazaleh \\ Department of Computer Science, Binghamton University \\ \{wyang,nael\}@cs.binghamton.edu
}

\begin{abstract}
Peer-to-Peer $(P 2 P)$ systems have become popular over the past few years. However, their large scale and the open nature of the system makes studying them challenging. This paper presents an extensible framework for simulating P2P networks efficiently and accurately. Efficiency is accomplished by using message level simulation rather than packet level simulation. Moreover, accuracy is maintained by tracking the network infrastructure and using a flow model to accomplish accurate estimate of the message behavior. A second contribution of the paper is to model the BitTorrent (BT) protocol. BT is a widely-used protocol that is significantly more complex than other P2P protocols because file download occurs in chunks from many other peers concurrently. Thus, contrary to models of other P2P systems such as Gnutella or Freenet, which focus on finding the location of a file in the network, BT's complexity occurs in downloading files (locating files in fact occurs out of band using websites that host the Torrent files). We validate the model against a packet level simulator and also using a real, but small scale, BitTorrent experiment. The simulator is object oriented and extensible for simulating other P2P protocols and applications.
\end{abstract}

\section{Introduction}

In recent years, Peer-to-Peer $(\mathrm{P} 2 \mathrm{P})$ networks have gained increasing popularity. As a result, the significant challenges that arise in their design and operation have been brought to light. Most recent $\mathrm{P} 2 \mathrm{P}$ research has focused on providing efficient lookup systems that can be used to build more complex systems; however, the behavior of these P2P systems under large scale and complex interactions is still poorly understood and many challenges in improving their performance remain. Unfortunately, their open nature and large

*This work was partially supported by NSF grant CNS-0454298 and AFRL grant FA8750-04-1-0211. scale makes it difficult to study their performance or to evaluate new ideas under realistic conditions.

The first contribution of the paper is a new simulation framework, the General Peer-to-Peer Simulator (GPS) [10], capable of accurate modeling and efficient simulation of $\mathrm{P} 2 \mathrm{P}$ protocols and applications. An important feature of GPS is that it supports modeling the download component which is commonly abstracted away in P2P simulators. Given the large sizes of files and the complexity and size of the underlying network, modeling file downloads is complex. However, GPS maintains efficiency by modeling communication at the message level. On the other hand, accuracy is maintained by taking into account the underlying network and protocol properties (in this case TCP) without the overhead of packet level simulation; this is achieved by the use of macroscopic models of estimating performance (e.g., [14]). The GPS framework provides all the infrastructures required for $\mathrm{P} 2 \mathrm{P}$ simulation, so new protocols can be easily plugged in and even run on existing protocols. Section 2 compares GPS to other P2P simulators. Section 3 overviews the design of GPS.

The second contribution of the paper is modeling the BitTorrent (BT) protocol $[4,7]$. BT is a widely-used protocol that employs a novel algorithm for distributing the file-sharing load among peers in a way that ensures participation. The basic idea in BT is to divide a single large file into small pieces (a typical piece size is $256 \mathrm{~KB}$ ). Peers query a centralized server, which is called tracker, to retrieve peer list owning part or whole of the file they are interested in, and simultaneously download different pieces of the file from different peers. Thus, BT relies on centralized components for file search. In addition, it employs a moderation system to ensure the integrity of file data. Finally, participation is ensured using a tit for tat technique for download which prevents users from free-loading. Section 4 presents a quick overview of BT. Section 5 describes the extension of GPS to model BT (which we call BTSim).

Most existing P2P protocols and applications, such as Chord [20] and Gnutella [9] focus on the problem of how to find documents in a P2P network. In BT, file search is not a problem due to the use of centralized servers. Rather, 
the complexity and novelty of the algorithm is in the download process. Thus, a different type of simulator is needed that can accurately model the complex download process of protocols such as BT.

The download process is greatly influenced by the network properties such as delay, bandwidth and transport protocol behavior. In contrast, models for traditional P2P protocols use small search messages; these models typically do not model the underlying network in sufficient detail to evaluate the download process. Thus, GPS models the network and accounts for protocol effects to more accurately capture the download behavior without doing low level packet level simulation. Section 6 describes the networkspecific support in GPS. In Section 7, GPS is validated and evaluated using sample BT scenarios. Finally, conclusions and future work are presented in Section 8.

\section{Related Work - Existing P2P Simulators}

Traditional packet-level network simulators provide accurate low-level models of the network hardware and protocols but are too detailed to be effective in analysis of large scale P2P networks. For example, the ns2 simulator [2], is perhaps the most widely used networking simulator. In addition to being too detailed, another problem in adapting ns-2 to P2P simulation is the complexity and interdependency between its simulation modules (scheduler, core simulator models, protocol models, and application level models); this makes it difficult to extend the simulator with new models. The large variety of $\mathrm{P} 2 \mathrm{P}$ algorithms and protocols requires ease of modeling and extensibility. There are a number of existing $\mathrm{P} 2 \mathrm{P}$ simulators. In the remainder of this section we overview of some of these simulators and compare them to our work.

Narses: Like GPS, Narses [1] uses a flow-based model to avoid simulation of lower level details. Experiments show a 45 times speedup over ns2, while using only $28 \%$ of the memory. Narses computes the available bandwidth based on end node bandwidth without considering network and protocol effects. While this simplified model improves performance, it is not accurate. The simulated completion times maintain an average accuracy of $8 \%$ for for small scenarios. However, for a large file sharing network, with high volume data traffic, the accuracy can be expected decrease.

Packet-level P2P (PLP2P): PLP2P [11] overlays P2P models on an otherwise packet simulator such as ns2. It creates wrappers that translate P2P level events into commands to the underlying packet simulator. This approach suffers the overhead of packet level simulation. The authors argue that $\mathrm{P} 2 \mathrm{P}$ system performance can be impacted by the network characteristics to a large extent. In their experiments they vary the access bandwidth and the link delay on the backbone in order to represent different degrees of proximity among peers. Both of these parameters affect the average throughput of the TCP tunnels used for communication in their Gnutella network, but cannot be accounted for in a simulator like Narses. GPS attempts to provide support of network and protocol effects, without the complexity of full packet-level simulation.

$\operatorname{SimP}^{2}$ : $\operatorname{Sim}^{2}$ is a graph-based simulator for analysis of ad-hoc P2P networks [13]. The analysis is based on a non-uniform random graph model, and is limited to studying basic properties such as reachability and nodal degree.

Query Cycle Simulator (QCS): QCS [19] simulation proceeds in query cycles representing the time period between issuing a query and receiving a matching response. In addition, the authors analyze content distribution performance and peer behavior. However, the effect of bandwidth is modeled in a simple peer-based way (similar to Narses).

PeerSim: Peersim [12] is a Java based search framework that allows modeling of P2P overlay search algorithms. To allow for scalability and focus on self-organization properties of large scale systems, some simplifying assumptions have been made. These include ignoring the details of the transport communication protocol stack. Since we also focus on the performance aspects of the download process, we do not abstract away the network details completely.

GPS is distinguished from these works by the following: (1)It models the performance of the network with more accuracy by allowing the users to use macroscopic models that account for bandwidth, delay and the effects of TCP. Thus, it provides the efficiency of message-level simulation with significantly better accuracy than existing simulators; (2) It provides an extensible object oriented framework that allows modeling of alternative $\mathrm{P} 2 \mathrm{P}$ protocols, alternative network models, and alternative flow-level models; (3) It provides integrated network topology generation and visualization support; and (4) It provides simulation models for BitTorrent, which, to the best of our knowledge has not been modeled functionally before.

\section{GPS Simulator Design}

GPS is a message-level, event driven P2P simulation framework aimed at modeling $\mathrm{P} 2 \mathrm{P}$ protocols accurately and efficiently in a realistic and dynamic environment. For the purpose of portability, ease of development and extensibility GPS uses Java as the development and simulation language. To support accurate simulation GPS models the network topology and characteristics. GPS is a discrete-event, rather than time-driven, simulator. Instead of advancing the simulation time in fixed increments and processing events synchronously at each clock tick, processing and time advancement is triggered by the occurrence of events. 


\subsection{Overall Architecture}

The overall architecture of GPS is shown in Figure 1. Configuration files are used to specify the simulation scenario. This includes the resource allocation model (bandwidth limitations, document placement), the user actions, and physical network topology. GT-ITM [21] is currently integrated with GPS allowing representative topologies to be studied. GPS provides tools to generate resource allocation file and user action file. Freely evolving P2P networks have been shown to exhibit power-law network characteristics [18]. In BT simulation, a key additional factor is the distribution of the documents. Configuration files can be generated based on traces of real experiments or according to statistical distributions. For example, in our experiments we use uniform distribution for resource allocation and user actions, but the selection of the files to be downloaded are distributed according to the Zipf [22] corresponding to their popularity rankings.

\subsection{Simulation Framework Components}

We organize framework components into core components (the basic components implementing the simulation engine and base network and protocol models), special components to provide user support.

\subsubsection{Core Components}

The core components include Simulation Engine, Topology, Protocols and Agents. It also includes two protocol related classes: Documents and Keywords.

Simulation Engine:this is a discrete event simulation engine, with a global queue and an event scheduler. Events include user actions, simulator internal events, and messages passed between peers. The simulation loop proceeds by selecting the next earliest event in the queue, executing it to completion, and inserting any newly generated events in the queue in simulation time-order. One event may have more than one handler, which would be useful for some situations such as broadcasting. The scheduler is implemented using a heap structure for performance considerations, so that insertion and deletion times are in $O(\log (n))$ for $n$ events. We are considering implementing a more efficient data structure for the event queue (such as calendar queue) for further improvement of performance.

Topology: Topology is a global object which can be used to retrieve network related information, such as delay between any two given nodes. We follow the GT-ITM model of considering a Transit-Stub topology. Transit networks represent backbone networks while stub networks model edge/customer networks. Each connection has an associated bandwidth and delay. Three categories of connections are considered, between transit nodes, between transit nodes and stub, and with in stub. The bandwidth and delay can be set by connection group, or read from a bandwidth and delay matrix per individual connection. Note that this topology refers to the underlying network topology, and not a logical overlay topology.

Protocols: Protocols implement the core of the P2P systems; this includes the structure of the overlay and functions such as the search behavior and responses to user actions. $\mathrm{P} 2 \mathrm{P}$ organizations (such as centralized directory model, or distributed structured or unstructured P2P) can be modeled by extending this component. The BTSim simulation model is one extension of this Protocol module.

Agents: Agents represent endpoints where P2P messages are constructed or consumed. They are used in the implementation of protocols at various layers. The abstract class Agent exposes a message handling interface to the application. Each node can attach more than one agent, some for lower-layer protocols (e.g. Chord agent for file look up), and some for higher-layer protocols (e.g. to implement a cooperative P2P file system).

Documents: Documents are the basic elements for filesharing P2P applications. It includes basic information of the file such as size.

Keywords: Keyword is an abstract class provided for extensibility, since most $\mathrm{P} 2 \mathrm{P}$ protocols use keywords to search.

\subsubsection{Special Components}

For ease of operation and to allow visual monitoring of progress, a GUI is integrated in the GPS framework. Asynchronous logging is also provided to reduce simulation time.

GUI: The incorporated GUI in GPS can help users analyze network topologies, identify distinguished nodes, monitor the dynamic changes in simulation progress, and interpret the observed behavior. GPS can run from the GUI interface or from the command line for better performance.

Asynchronous Logging: Event logging is an expensive operation that leads to observable slowdown in the performance of the simulator. To reduce simulation time, GPS provides option for using a separate logging thread to provide asynchronous logging, thus the simulating thread does not have to block while writing log records.

\section{The BitTorrent P2P Protocol}

An archive $P$ is logically partitioned into fixed size (e.g. $256 \mathrm{~K})$ pieces that serve as the basic download unit in BT. To publish $P$ via BT, first a torrent file must be created and hosted on a website. Peers that are interested in downloading the file, must find the torrent file on the publishing website. The torrent file includes information regarding the 


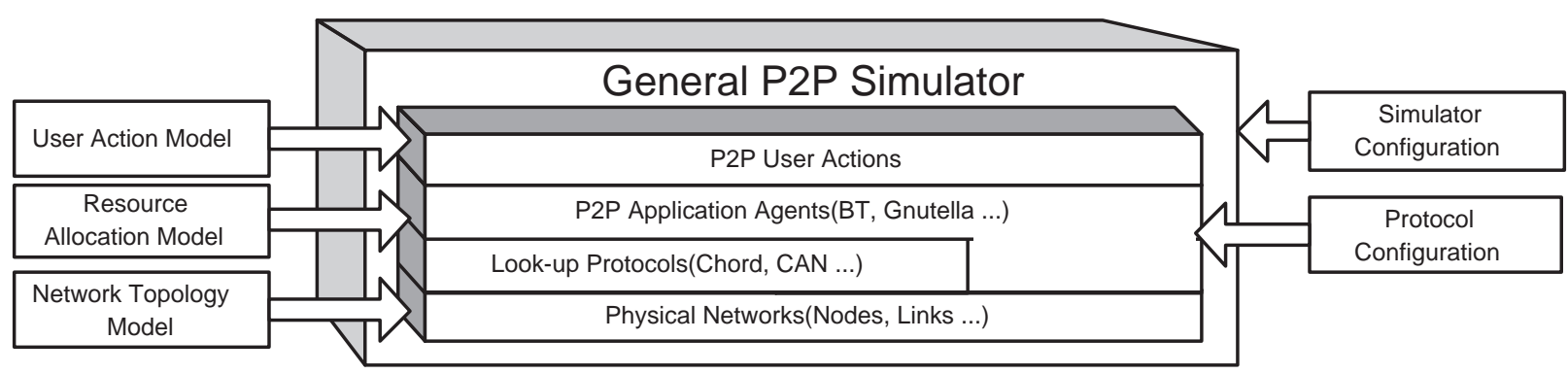

Figure 1. GPS Architecture

file, including the address of a tracker. The tracker is a non-content-sharing node in the BT network which serves as a coordinator for the download process for $P$. Peers include seeds which generously provide access to the file, and leechers who generally have already downloaded some of the file pieces and are trying to get the rest.

\subsection{Download Initiation and Basic Operation}

A peer that loads a torrent file in a a BT client first announces itself to the tracker. The tracker responds with a randomly selected subset of active peers. Thus, the tracker is the only rendezvous point for peers to discover each other. The peer then select a subset of the list to which it initiates a handshaking request to setup a bidirectional TCP connections. The peers proceed to continuously download pieces from other peers that can provide them according to the piece selection and choking algorithms described below.

If this is the first peer to attempt downloading the file, there will be only one seed (the original uploader) and the cost of uploading will be borne solely by this host. As the archive is disseminated among a growing number of connecting leechers, the percentage of replicated content increases, lowering the percentage of unique content on the original host. When multiple leechers are downloading the same archive at the same time, they upload pieces of the archive to each other. This redistributes the cost of upload to leechers, thus making hosting an archive with a potentially unlimited number of downloaders affordable. Once a leecher obtains a complete copy of the archive, it is promoted to a seed status and may remain on the network solely as an uploading node at the peer owner's discretion.

\subsection{Piece Selection and Peer Choking Algorithms}

Piece selection and choking are two major mechanisms in BT that make downloading efficient and that ensure that peers balance download and upload fairly. Piece selection allows a peer to determine which piece to download next.
Peers select a piece to download from a connected peer according to the following rule: first check unfinished pieces that are not currently being downloaded to attempt to complete them; if none exist, the rarest piece available (at peers among the peer list) is chosen first. Exceptions to this rule are selection at the beginning of the download, when a peer uses random selection, and near the end (the so called endgame) to try and get the missing pieces from anybody. Peers announce update other peers connected to them with their piece information to help make better selection decision.

Each peer attempts to maximizing its own interest by downloading as much as it can. Fairness is achieved because of the tit-for-tat approach on deciding uploads. Because peers that provide more upload are more likely to be allowed to download by others (via optimistic unchoking, described below), participation is maintained and fair operation results. Rechoking and optimistic unchoking proceeds as follows. Of a peer's many established TCP connections, a small subset $u$ is internally marked by the peer as being unchoked, and others are marked as candidates. An unchoked connection is one where requests from the other end for a piece will be fulfilled.

Each peer periodically chokes a fixed number of peers (e.g. 4). The decision on which peers to choke/unchoke is solely based on dowloading/uploading rate, which is evaluated on a rolling average. Seeds unchoke $k$ peers with the highest downloading rate from them, and leechers unchoke $k$ peers with highest uploading rate to them. This process is called a Re-choke. This algorithm sieves out the slower peers, allowing them to connect to others with comparable speeds, and allows those with more bandwidth to be connected to peers that can help maximize that bandwidth

In order to discover unused connections that are better than the ones being used, both seeds and leechers unchoke one peer regardless of the download/upload speed, rotating every third re-choke period - this is called Optimistic Unchoke. It is possible for a peer to get simultaneously choked by all peers it is connected to. To deal with this situation (called snubbing), a BT peer assumes it is snubbed when 60 seconds pass without receiving any data from a given peer, 
and will initiate several concurrent optimistic unchokes.

\section{BTSim Simulation Components}

We used the framework to model the BitTorrent protocol completely as per the BT specification $[3,5]$. We call the resulting model BTSim. Other protocols can be modeled in a similar fashion. In this section, we overview the BTSim components.

BT Protocol: BT protocol is an instance of Protocol in the framework. The agent attachment, document allocation, and user action registration are done in this class.

BTTrackers and BTPeers: BTTracker and BTPeer are instances of Server and Peer, which in turn are descendents of Agent. BTTracker processes HTTP GET messages, and BTPeer processes tracker response and peer messages using the event handler in Agent.

BTDocuments: In addition to regular document properties, BTDocument provides more specific information required by BT (e.g., piece information). BTDocuments also include popularity information clue for random downloading.

BTSessions, BTSockets and BTConnections: A BTSession is responsible for downloading and/or uploading of one BTDocument. Each BTSession includes several BTConnections between BTPeers. BTPeers open a BTConnection through BTSockets at each side. One BTConnection stores bandwidth information for both directions. BTSockets store connection information at each end.

BT Algorithms: All algorithms such as peer selection, piece selection, recoking, and optimistic unchoking, are implemented as separate modules. They can be extended or replaced to investigate algorithmic optimizations to BT.

\section{Accounting for the Network and Protocol Effect}

In packet level simulation, bandwidth is managed by calculating the transferring time of each packet, including modeling of the effect of queues. Network protocols are modeled and govern when packets are sent and how they react to packet losses. This approach can provide tremendous accuracy, but requires high simulation time. Approximate models may be sufficient, especially when we are interested in macro-level phenomena such as average document download time. In contrast, message level simulation considers application level message transfers while abstracting away the details of the network. Ignoring network and protocol effects leads to inaccurate estimates of the message transfer time.

GPS attempts to address these limitations by providing a more accurate link-based macro-model (or flow-level) estimate of the message transfer time. GPS provides a selection of flow models, and mechanisms to allow users to add additional flow models if they so desire. Thus, protocols that are sensitive to the flow characteristics (such as BT) can use more accurate models, while other protocols that study, for example, the effectiveness of search algorithms are free to use more efficient, simpler models. In the remainder of this section, we describe the supported flow models.

\subsection{Peer Based Bandwidth (PBB) Flow Model}

Most message level simulators adopt a simple model of available bandwidth if they do bandwidth management: Bandwidth is assigned to a peer rather than to a link. This choice is made because calculating the maximum bandwidth for a single peer is much easier than tracking the whole network (including modeling cross traffic from other applications). Moreover, this model does not account for the effects of the communication protocol (e.g., TCP), and assumes that the connection is able to grow immediately to take up the available bandwidth.

\subsection{Dynamic Link-level Network Bandwidth (DLNB) Flow Model}

DLNB (which is proposed in this paper) extends PBB to account for bandwidth limitations that occur in the middle of the network. Bandwidth calculation is not based on peers only, but also on links from the Transit-Stub model and connections in $\mathrm{P} 2 \mathrm{P}$ applications. A flow represents a large message, which consumes relatively large transmission time: for example, a flow could be the $256 \mathrm{~KB}$ block peer message in BT. The message is typically passed on from the application to the transport layer to send to a recipient. Other peer messages in BT are considered to be only affected by delay but not by bandwidth due to their small size.

Bandwidth is allocated initially per configuration and consumed when connections are established. Upon uploading or downloading a file, peers try to use maximum bandwidth along links on the connection. The bandwidth achieved by connections on a link is not always equal (e.g., if both are using TCP, the bandwidth share is known to be inversely proportional to the connection RTT). Unlike the problem of finding the all-pairs shortest path, or minimum spanning tree, where commonly used algorithm can be adopted, finding the maximum possible bandwidth of a connection between two Agents, and managing bandwidth allocation on each Link does not have existing known algorithms. Therefore, we develop the following algorithms to track the available bandwidth on links.

Algorithm for Bandwidth Allocation:

Step 1. Find the maximum available bandwidth on each link along the connection. If the available bandwidth on any 
link is less than the average bandwidth across all active connections on the link after this connection is allocated, adjust other connections on that link to make sure this connection can get an equal share of the bandwidth.

Step 2. Find out the minimum negotiated bandwidth along the connection, and allocate to all links of the connection.

Algorithm for Bandwidth Deallocation:

Step 1. Release occupied bandwidth to available bandwidth on each link along this connection.

Step 2. Find out bottleneck along this connection, i.e. link with minimum available bandwidth.

Step 3. Relax all connections on that link, i.e. let every connection on this link try to allocate it's maximum possible bandwidth using allocation algorithm.

Step 4. Find bottleneck, and relax again, until find same link as bottleneck twice.

\subsection{TCP Based Flow (TBF) Model}

TBF assumes that TCP is limited by the error/congestion rate and not the capacity of the links. Further, it assumes that the connection is unsaturated. For these reasons, we believe that there is room for additional macro models $[14,16,17,6,15]$ that take into account the underlying network characteristics and TCP characteristics to provide an estimate of the expected bandwidth of the connection. In this paper, we only investigate one of these models [14]. In this model, the available bandwidth is estimated per the following equation.

$$
B W=\frac{M S S}{R T T} \frac{C}{\sqrt{p}}
$$

where $B W$ is the available bandwidth, $M S S$ is the maximum segment size used by $\mathrm{TCP}^{1}, C$ is a constant that depends on the TCP flavor used (more specifically, whether it uses immediate or delayed ACKs), and $p$ is the estimated packet loss rate on the connection (typically due to congestion).

\section{Simulation Results}

In this section, we present our experimental methodology and give preliminary results using GPS simulator. We also present preliminary validation of the flow model (by comparing to a packet level simulator) and the simulator (by comparison to a small scale real BT experiment). The performance and scalability of GPS are also studied.

\footnotetext{
${ }^{1}$ MSS is typically computed after path Maximum Transfer Unit (MTU) discovery discovers the maximum packet size that will not be fragmented. TCP MSS is then the size of the discovered TU after subtracting the size of the TCP and IP headers.
}

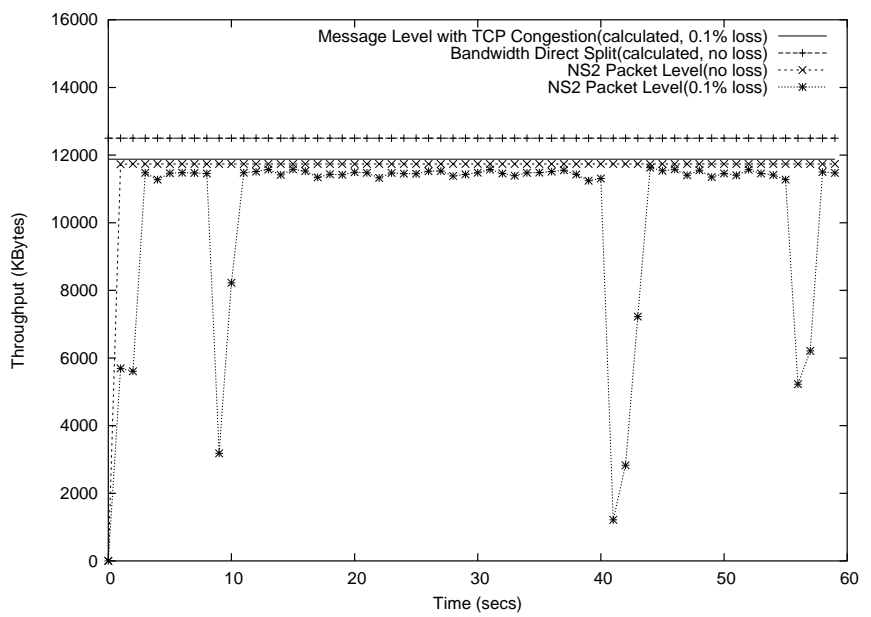

Figure 2. Packet and Message Level Comparison

\subsection{Macro Model Simulation Validation}

We evaluate the accuracy of message level simulation by comparing the calculated TCP throughput using TBF model with packet level simulator simulated throughput. We consider two nodes connected by a link with $100 \mathrm{Mbps}$ bandwidth and $0.1 \mathrm{~ms}$ delay. We used the widely used Network Simulator ns-2 [2] to simulate: (1) no loss situation, (2) $0.1 \%$ loss model, and compare them with calculated macroscopic TCP throughput using formula provided by [14].

MSS is the maximum segment size; we use ns2's default MSS of 536 bytes. RTT is round trip time, we use $1.7 \mathrm{~ms}$, which comes from observing the RTT time obtained via ns-2. A C value of 1.22 is used (as suggested for delayed ack TCP in the original paper [14]) and p of $0.1 \%$, also as recommended by the paper. The comparison is shown in Figure 2.

While some divergence between the models can be observed, they perform fairly similarly. Thus, we believe the small loss in accuracy is worth the savings in simulation time obtained by using the macroscopic flow models. Moreover, we believe that the parameters can be chosen more effectively to make the two models closer; specifically, a better methodology for selecting $p$ may be necessary.

\subsection{Link Based Flow Model Simulation Validation}

In this study, we validate GPS against a small-scale but real BT download experiment on the same topology. For our simulations we use a GT-ITM 10 nodes Transit-Stub topology; the topology mirrors the environment where we 


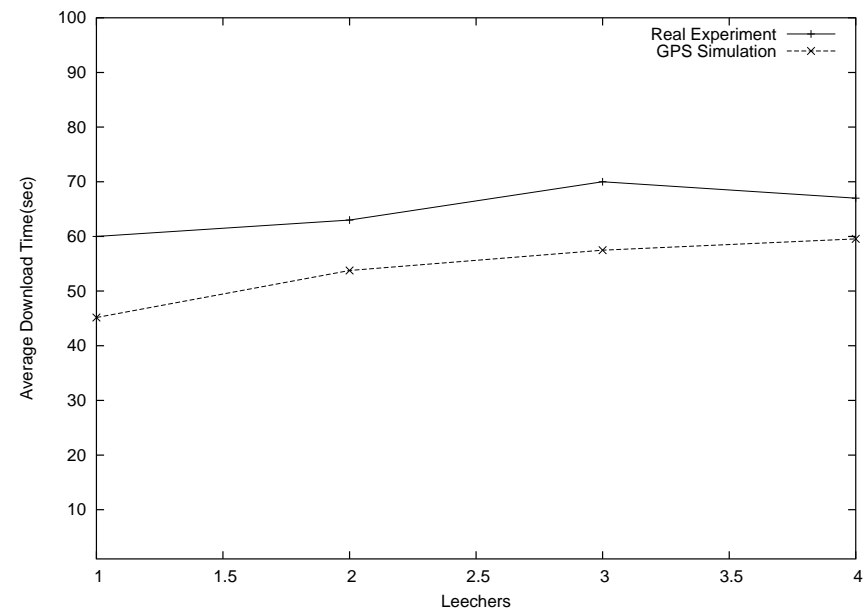

Figure 3. Average Download Time Validation

run the BT experiments. This simulation uses the DLNB model.

The nodes used in the experiment are all $2.0 \mathrm{GHz}$ Xeon SMPs with $1 \mathrm{~GB}$ memory running Debian Linux. They are connected to each other with $100 \mathrm{Mbps}$ switched ethernet network. All nodes are connected through a Transit node, which is an Ethernet switch in the real environment - all the nodes are symmetrically positioned in terms of network topology. The measured delay was approximately $0.1 \mathrm{msec}$. The size of the file used in the experiments is $526.58 \mathrm{MB}$.

Figure 3 shows comparison of real experiments and the simulation. In this example, one node is a BT tracker, one is a seeder, and other nodes are leechers that send requests to the tracker for download. We compared the average finish time for the four leechers. The real experiment was conducted using the official BT client from [4]; the only change made to this client code is to remove the $20 \mathrm{~KB} / \mathrm{sec}$ upload rate restriction to exercise the network model and to ensure that the experiments finish in reasonable times. The figure shows that the simulation model and the experiment are fairly close to each other.

Running BT on a LAN may not be a representative situation, but due to firewall restrictions in our testbed we use this setting. We hope to validate against experiments conducted using hosts with different points of attachment to the Internet in the future.

\subsection{Scalability Analysis}

In this experiment, two-level Transit-Stub topologies are generated using GT-ITM. Bandwidth between transit nodes is set to $1000 \mathrm{Mbps}$, between transit and stub is $100 \mathrm{Mbps}$, with no connections within stubs. Delay between transit nodes is set to $5 \mathrm{~ms}$, and $10 \mathrm{~ms}$ between transit and

\begin{tabular}{|r||r|l|l|l|}
\hline & Nodes & Peers & Documents & Downloads \\
\hline 1 & 16 & 8 & 4 & 4 \\
\hline 2 & 64 & 32 & 16 & 16 \\
\hline 3 & 252 & 128 & 64 & 64 \\
\hline 4 & 1054 & 512 & 256 & 256 \\
\hline
\end{tabular}

Table 1. Simulation Scale

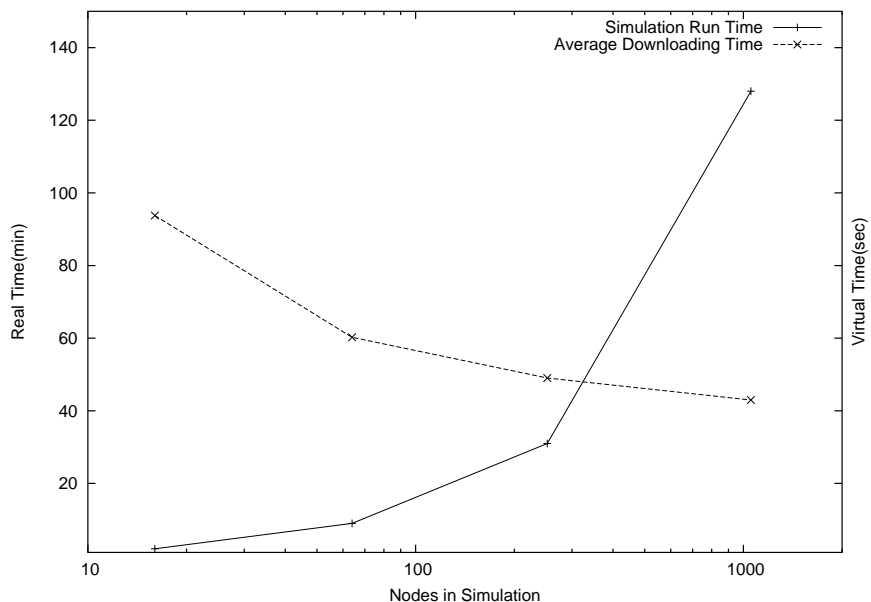

Figure 4. Simulation Scalability Study

stub nodes. BT Peers are randomly attached to non-transit nodes; Documents with different popularity rankings are randomly stored at Peers; User actions (downloading) are randomly initiated every 50 seconds from the beginning. When a random download action is triggered, the downloading peer selects one document to download based on popularity ranking from trackers document database according to Zipfian distribution. All the documents are $500 \mathrm{MB}$ in size. Only one tracker is used randomly attached to a non-transit node in each topology. The size of the experiment is scaled according to Table 1 .

The result are shown in Figure 4. The simulation run time increases from 2 minutes 13 seconds to 128 minutes, and the average download time for each document drops by more than half. This result is consistent with the observation that download times drop with a larger number of peers (due to the Zipfian distribution of requests).

During the simulation, we noticed an interesting effect where the peers who join the downloading process later gradually caught up with the earlier peers. This may be caused by the piece selection algorithm, i.e. the latter started peer has less chance to fail to pick a piece to download, but more chance to find another peers with spare uploading bandwidth. We confirmed this effect using experiments as well. We also confirmed the effect of piece pipelin- 
ing, a known optimization to BT. BT is an Internet overlay application, so delay between peers are usually large. To compensate for the round trip delay between two consequent requests, request pipelining is introduced. Using pipelining, the request for the next block is sent while the current block is being downloaded. Thus, when the first block finishes downloading, the sender can immediately send the second block without waiting for the subsequent request significantly improving performance.

\section{Conclusion and Future Work}

In this paper, we presented and validated a P2P simulator with several desirable properties. Like most existing P2P simulators, GPS is efficient because it uses macro-models to abstract detailed network behavior. However, unlike these simulators, GPS incorporates a description of the network, and allows the users to select from a menu of macro models that incorporate the network and protocol properties more effectively. GPS has several other desirable properties, including extensibility to model any $\mathrm{P} 2 \mathrm{P}$ protocol, integration with a GUI and network visualization, and integration with topology generation tools.

The second contribution of the paper is to develop a model of BT that is faithful to the protocol specification. To our knowledge this is the first BT simulator that models the full behavior of the protocol. The simulator can help in understanding the behavior of this dynamic and complex protocol, and provides an ideal vehicle for evaluating optimizations to it. We are currently pursuing such optimizations such as geographical peer selection and the use of bloom filter summaries. From the evaluation, we show the validity of the use of macro level models in the simulation by comparison against a packet level simulator. Moreover, we validate the BT model by comparison to a small scale BT experiment.

Our future work targets continued improvement of the simulator. We would like to explore more accurate flow level models available in the networking community [16, $17,15]$. Other P2P protocols will be added to the framework. We will also consider multi-threading/parallelizing the simulator [8] in the future. Also, to achieve higher runtime efficiency, it is possible to compile GPS to native code, or alternatively, develop a $\mathrm{C}++$ version of the simulator. We hope to develop models for additional protocols with the assistance of others that may be interested in GPS.

\section{References}

[1] M. Baker and T. Giuli. Narses: A Scalable Flow-Based Network Simulator. Technical report, Department of Computer Science, Stanford University, CA, Nov. 2002.
[2] Berkeley/LNBL/ISI. The NS-2 Network Simulator. http://www.isi.edu/nsnam/ns/.

[3] Official bt 2005. http://www.bittorrent.com/protocol.html.

[4] Bt official website, 2005. http://www.bittorrent.com/.

[5] Wiki bt specification, 2005. http://wiki.theory.org/BitTorrentSpecification.

[6] A. Chaintreau and D. DeVleeschauwer. A closed form formula for long-lived tcp connections throughput. Perform. Eval., 49(1-4):57-76, 2002.

[7] B. Cohen. Incentives to Build Robustness in BitTorrent, May 2003. http://bitconjurer.org/BitTorrent/bittorrentecon.pdf.

[8] R. M. Fujimoto. Parallel discrete event simulation. Commun. ACM, 33(10):30-53, 1990.

[9] Gnutella, 2005. http://gnutella.wego.com.

[10] General p2p simulator, http://www.cs.binghamton.edu/ ${ }^{\text {'wyang/gps. }}$

2005.

[11] Q. He, M. Ammar, G. Riley, H. Raj, and R. Fujimoto. Mapping Peer Behavior to Packet-level Details: A Framework for Packet-level Simulation of Peer-to-Peer Systems. In Proceedings of MASCOTS 2003, Orlando, FL, Oct. 2003.

[12] M. Jelasity, A. Montresor, and G. P. Jesi. Peersim Peer-toPeer Simulator, 2004. http://peersim.sourceforge.net/.

[13] K. Kant and R. Iyer. Modeling and Simulation of Adhoc/P2P Resource Sharing Networks. In Proc. of TOOLS'03, Nov. 2003.

[14] M. Mathis, J. Semke, and J. Mahdavi. The macroscopic behavior of the tcp congestion avoidance algorithm. SIGCOMM Comput. Commun. Rev., 27(3):67-82, 1997.

[15] D. M. Nicol and G. Yan. Discrete event fluid modeling of background tep traffic. ACM Trans. Model. Comput. Simul., 14(3):211-250, 2004.

[16] J. Padhye, V. Firoiu, D. Towsley, and J. Kurose. Modeling tcp throughput: a simple model and its empirical validation. In Proceedings of SIGCOMM '98, pages 303-314, New York, NY, USA, 1998. ACM Press.

[17] J. Padhye, V. Firoiu, D. F. Towsley, and J. F. Kurose. Modeling tcp reno performance: a simple model and its empirical validation. IEEE/ACM Trans. Netw., 8(2):133-145, 2000.

[18] M. Ripeanu and I. Foster. Mapping the Gnutella Network Macroscopic Properties of Largescale P2P Networks. IEEE Internet Computing Journal, 6(1), 2001.

[19] M. T. Schlosser, T. E. Condie, and S. D. Kamvar. Simulating a File-Sharing P2P Network. Technical report, Department of Computer Science, Stanford University, CA, 2002.

[20] I. Stoica, R. Morris, D. Liben-Nowell, D. R. Karger, M. F. Kaashoek, F. Dabek, and H. Balakrishnan. Chord: a scalable peer-to-peer lookup protocol for internet applications. IEEE/ACM Trans. Netw., 11(1):17-32, 2003.

[21] E. W. Zegura, K. L. Calvert, and S. Bhattacharjee. How to model an internetwork. In IEEE Infocom, volume 2, pages 594-602, San Francisco, CA, March 1996. IEEE.

[22] H. Zipf. Human behavior and the principle of least effort. Addison-Wesley, 1949. 